\title{
Supporting Request Acceptance with Use Policies
}

Thomas C. King, M. Birna van Riemsdijk, Virginia Dignum, and Catholijn M. Jonker

TU Delft, Delft, The Netherlands

\{t.c.king-1, m.b.vanriemsdijk, m.v.dignum, c.m.jonker\}@tudelft.nl

\begin{abstract}
This paper deals with the problem of automating the contribution of resources owned by people to do work for others, whilst providing a means for owners of resources to maintain autonomy over how, when and to whom their resources are used with the specification of resource use policies. We give representations of requests for resource usage as a set of conditional norms, and a use policy as specifying what norms should and should not be imposed on a resource, i.e. a set of metanorms. Our main contribution is a reasoner built on the Event Calculus, that takes a request and a use policy and determines if the request is accepted, whether it would would conflict with the use policy.
\end{abstract}

\section{Introduction}

Increasingly, detailed environmental data is needed to inform the decisions of citizens and governments that are affected by environmental conditions. For example, smart cities' early warning flooding systems, or the choice of transportation based on the weather. Yet, it is not always feasible to analyse the type of data required and then undertake a costly and non-flexible deployment of a high-density sensor network.

To this end, crowdsensing (crowdsourced mobile sensing [8]) can be leveraged to obtain fine-grained, weather-specific, just-in time information about environmental conditions. For example, monitoring the rain levels around a crucial part of city water infrastructure by making ongoing agreements with a large number of citizens to collect and send rain data with their cellphones whenever they are in the area.

However, owners of existing sensor resources, such as rain sensors on bicycles communicating via cellphones, will not want them to serve all requests. Owners will want to maintain autonomy over their resource by specifying use policies governing how, when and to whom their resource serves requests. For example, owners of resources could specify that they do not want to serve requests that prohibit them from turning their device off, or require serving untrusted people. Furthermore, a resource owner can have requirements to be met for the use of their resource, such as payment.

Since a request expresses what the requestor wants the provider to do and not do, we represent a request as a set of norms. A use policy then governs a resource 
by specifying what norms ought and ought not be entailed by a request. Thus a use policy specifies norms about norms, i.e., we introduce the idea of specifying a use policy as a set of meta-norms.

In this paper we propose a reasoning mechanism that allows an agent that governs a resource (the provider agent) to determine what conflicts there are between a request and the use policy placed on the resource. The advantage of using an agent to govern a resource is that most requests can be handled automatically. We model the reasoner using the Event Calculus, allowing us to express the effect of events on instancing and directing norms and meta-norms at agents (detachment [1]) and terminating norms and meta-norms, thereby detecting conflict in particular circumstances.

After discussing related work (Section 2), we give an overview of our approach and its architecture (Section 3). Then, we introduce the specification languages for requests and use policies (Section 4) and their informal meaning. We specify the formal semantics using the Event Calculus [11] in Section 5. We validate our reasoning system with a formalisation of a motivating example in Section 6. We conclude the paper and discuss future work in Section 7.

\section{Related Work}

Our proposal fits into the broad area of normative multi-agent systems (see [1] for a recent literature survey), the formal study of which is deontic logic (see [7]). Much work has been done on reasoning about normative agreements such as contracts and compliance with norms (e.g. $[4,9,15,17,18])$ and multi-agent organisational frameworks for the verification of organisations as networks of contractual agreements (e.g. $[6,10])$.

However, we focus on a pre-agreement stage, where the novelty of our proposal is the application of meta-norms to govern resource use, with a logic programming implementation for reasoning about conflicts between requests and use policies. We find the two most closely related areas to our aims are on metanorms, and on the formalisation of normative frameworks in the Event Calculus.

López et al. [12] propose legislative norms to govern which norms an agent may issue or abolish. These are a type of meta-norm, but whilst they conceptually define which norms can be introduced into the system and when, they do not provide an implementation level mechanism for checking meta-norm/norm conflicts based on their operational semantics as we do.

Boella and Torre [3] propose a kind of permission as a meta-norm issued by a higher authority to block the imposition of norms by lower level authorities. Using input/output logic (a logic of conditional norms [13]), their formalism produces for a given situation what may be obliged by lower-level authorities given everything that is permitted by higher-level authorities. The main difference with our work is that they give permissions the role of derogation and do not consider obligatory meta-norms. Our work is also different because we define operational semantics as a logic-program, making it straight-forward to implement our proposal. Furthermore, unlike in the aforementioned work, we do this 
for norms with deadlines which affect if they can be simultaneously detached with meta-norms and thus cause conflict.

Meanwhile, Wansing [16] treats both obligatory and prohibitory meta-norms in deliberative-stit logic [16] as being norms about the action of imposing norms. The work is done in a setting where there is a hierarchy of authorities, so for example, one authority obliges a lower authority to forbid an even lower authority. Like Wansing, we define a meta-norm as being about the event of a norm being imposed. We also explicitly represent norms as being from one authority to another, and meta-norms are implicitly so. But again, our work is different in that it contributes a meta-norm/norm conflict detection mechanism, where norms can be both conditional and with deadlines, as a logic-program.

Finally, we define our operational semantics using the Event Calculus, a logic programming formalism. Thus, there is also related work that applies the Event Calculus to modelling normative systems. Some of this work includes $[4,17,18]$, where social commitments (norms bound to agents as a part of an agreement) are modelled using the Event Calculus, allowing the delegation of existing commitments and the modelling of contract lifecycles. Unlike our proposal, the aforementioned work does not consider the possibly conflicting relationship between meta-norms and norms.

\section{Overview}

Throughout the paper we consider a scenario where a municipality wants to know detailed rain statistics near a newly built water square (an above-ground area that is both a recreational square, and a place to store rain water temporarily until there is capacity in the sewage system to handle the water ${ }^{1}$ ).

Assuming there is no dense network of stationary rain sensors in the area, the municipality might opt to make an ongoing agreement with the mobile sensors of people that frequent or pass by the square, such that whenever they are near the square they will collect and send rain data. For example, by recruiting users with an app on their mobile phone that communicates with rain sensors on their umbrella or bicycle to collect rain data, such that the sensors transmit the rain data they gather to their mobile phones which then send it to the requestor.

For simplicity we consider a process between two agents, depicted in Figure 1. These are, the resource provider agent governing a resource owned by a user and a requestor agent used by someone who wants to request use of the resource. The process begins with the user wanting data specifying the terms of the request, using the lexicon of a common ontology describing events, which their requestor agent then poses to the resource provider agent. The owner of the resource has specified the terms of its use policy, using the same common ontology of events.

The resource provider agent reasons over the use policy and request and detects conflict between the two. This supports the automated governance of

\footnotetext{
${ }^{1}$ http://www.raingain.eu/en/actualite/rotterdam-inaugurates-first-large-scalesquare-water-storage-greenery-and-sport
} 
resources, by directing the resource provider agent to refuse requests that conflict with the use policy.

There are two types of conflict to consider. The first type of conflict occurs if the request does not impose a norm in circumstances where the use policy obliges it to be imposed E.g. an obligation to oblige a requestor to provide payment. The second type of conflict occurs if the request would impose norms in circumstances where they are prohibited by the use policy, for example, a prohibition on prohibiting the free movement of a resource.

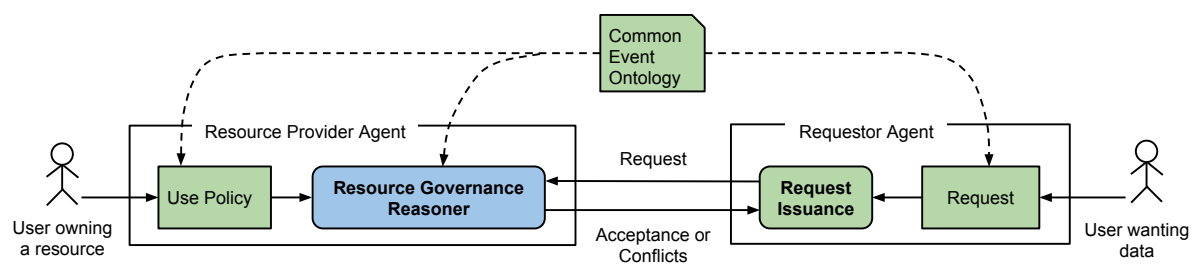

Fig. 1. Overview

In this paper, we focus on the reasoning about requests for resources governed by use policies, including the representations of requests and use policies. An overview of the resource governance reasoner architecture is given in Figure 2.

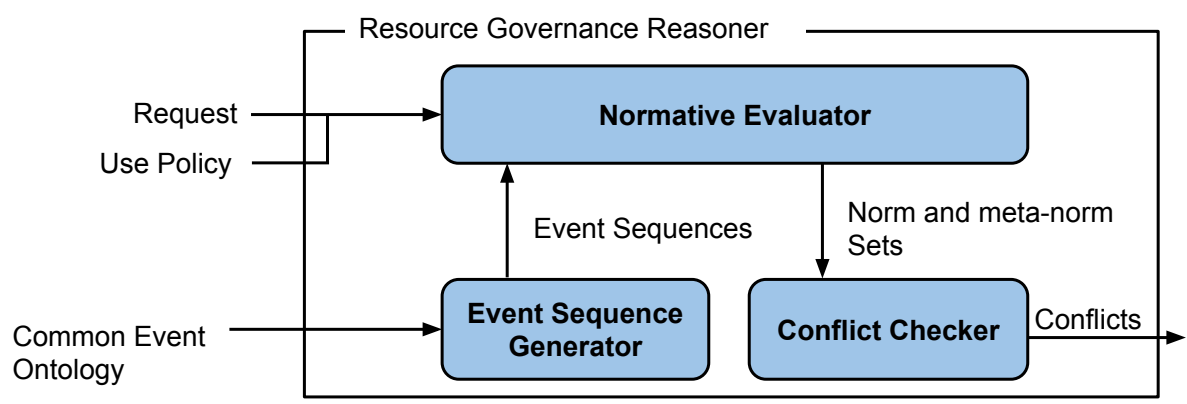

Fig. 2. The Architecture of the Reasoner

The resource governance reasoner we propose takes as inputs an ontology of events (what can happen), a request, and a use policy. The ontology of events is used to generate a sequence of events for the normative evaluator, in order to determine under which circumstances (after which events) which norms and meta-norms are detached simultaneously. Simultaneously detached norms and meta-norms are output as sets for the conflict detector. As a result, the reasoner returns the conflicts between a request and a use policy. 
We model norms and meta-norms, detached and terminated according to a sequence of events, by using the Event Calculus [11] as the underlying formalism.

\section{Requests and Use Policies}

In this section we introduce the languages of requests as a set of norms and use policies as a set of meta-norms (4.1), then we proceed to discuss how norms and meta-norms relate to each other in terms of conflicts (4.2).

\subsection{Representations}

We use requests to represent what a requestor would like a provider to agree to doing and a use policy to govern what a provider agent will agree to. We begin with a motivating example to motivate our representations of requests.

Example. (Part One, Requestor) Rachel wants to monitor the level of rainfall around a newly built water square. There is no dense network of static sensors, so she wishes to make use of the existing mobile sensors people carry to gather the data. Thus, she requests people to make an ongoing agreement to provide her with precipitation data. The terms of the request are that whenever someone enters the water square, rain data should be gathered, once data is gathered it should be sent before the sensor leaves the area around the station, and the sensor should not be turned off until data has been gathered.

Norms concern agents, so we assume a set of agent names Ag. Norms are about events, which can be generated by or concern specific agents (e.g. an agent entering an area), or the environment (e.g. rain starting to fall). Thus, events are either non-agentive, denoted as propositions, or agentive denoted as propositions with an agent name in the subscript.

Definition 1. (Events) Let EnvProp with typical element EP, and AgProp with typical element AP be mutually disjoint sets of propositions, respectively denoting non-agentive and agentive events. Also let $\mathrm{Ag}$ be a set of agent names with typical element $a$. The set Ev is the set of all elements ev expressible in the language:

$$
e v::=E P \mid A P_{a}
$$

Norms are obligations and prohibitions, respectively denoted with a deontic type of $O$ or $F$. Norms oblige or prohibit an agent to ensure an aim $A$ happens (an event) possibly before a deadline $D$ (also an event).

A norm can be detached (become active) [1], either unconditionally or on the condition of an event happening. If a norm's detachment is conditional on an event, we say it is a conditional norm, otherwise it is an unconditional norm. Conditional norms are represented as a rule with the conditional event $C$ placed in the antecedent and a norm as the consequent.

Finally, we follow the notion that when a norm is detached it is imposed on a debtor denoted with $D E$, towards a creditor denoted with $C R$. For example, 
a debtor can be an agent obliged to provide data within one minute towards a creditor requesting it, or a debtor can be an agent obliged to pay for data towards an agent providing the data.

Definition 2. (Norm) Let $O$ and $F$ respectively denote the deontic types of obligation and prohibition, $D E, C R \in A g$ be debtor and creditor agents, and $C, A, D \in E v$ be events respectively denoting the condition, aim and deadline of the norm. An unconditional norm is denoted with $\langle u c n\rangle$ and a conditional norm is denoted with $\langle c n\rangle$. The set of norms $N$ is the set of all elements $\langle n\rangle \in \mathcal{N}$ expressible in the language defined as:

$$
\begin{aligned}
\langle n\rangle \quad::= & \langle c n\rangle \mid\langle u c n\rangle \\
\langle c n\rangle::= & C \text { THEN }\langle n\rangle \\
\langle u c n\rangle::= & O_{D E: C R}(A \text { BEFORE } D) \mid F_{D E: C R}(A \text { BEFORE } D) \mid \\
& O_{D E: C R}(A) \mid F_{D E: C R}(A)
\end{aligned}
$$

A request, such as Rachel's, is simply a set of norms.

Definition 3. (Request) $A$ request $R$ is a set of norms such that $R \subseteq N$.

Thus we can formalise the request $R$ of Rachel $(r a)$, to a resource owned by a person called Peter ( $p e)$, as:

$$
\begin{aligned}
& R=\left\{\text { enter_water_square } e _ { p e } \text { THEN } O _ { p e : r a } \left(\text { gather_rain_data } a_{p e}\right.\right. \\
& \text { BEFORE send_data } \left.a_{p e}\right) \text {, } \\
& \text { enter_water_square }_{p e} \text { THEN } O_{\text {pe:ra }}\left(\text { send_data }_{p e}\right. \\
& \text { BEFORE leave_water_square } \left.{ }_{p e}\right) \text {, } \\
& \text { enter_water_square } e_{p e} \text { THEN } F_{p e: r a}\left(\text { sensing_off } f_{p e}\right. \\
& \text { BEFORE gather_rain_data } \left.\left.{ }_{p e}\right)\right\}
\end{aligned}
$$

Use policies are used to govern a resource, such that requests a resource owner does not want to be automatically accepted will be detected as conflicting with the request, and rejected. We use the following example to motivate the expressivity of our use policy representation.

Example. (Part Two, Provider) Peter has a cellphone app that can measure rainfall in a location by communicating using bluetooth with a sensor on his umbrella. Peter usually carries his cellphone and umbrella with him, including when he walks past the water square. He is willing to donate the use of his sensing resource, but only if he does not have to stay in any particular area. His cellphone uses up a lot of energy when collecting data, so he wants to be allowed to turn the sensing off when the battery's energy becomes low. Finally, if he is obliged to provide data, then he wants to be paid within one day. 
We represent a use policy as a specification of what norms should and should not be detached under specific circumstances. That is, it is a set of norms about norms, or meta-norms. This specification can be compared against a request.

Meta-norms can be conditional on an event or a norm being detached. For example, on the condition an obligation for data to be provided is detached, then it is obligatory for the agent that should be sent data to provide payment.

An unconditional meta-norm umn is detached by default, whilst a conditional meta-norm $\mathrm{cmn}$ is detached on the condition an event $C$ occurs, or a norm $n$ specified in the condition is detached. Both conditional and unconditional metanorms obligate or prohibit the detachment of a norm $n$. For brevity we do not examine the case where a meta-norm has a deadline, but our framework can easily be extended to accommodate for this.

Definition 4. (Meta Norms) Let $n, n^{\prime} \in N$ denote norms, and $C \in E v$ be an event. An unconditional meta-norm is denoted with $\langle u c m n\rangle$ and a conditional meta-norm is denoted with $\langle c m n\rangle$. The set $M N$ is the set of all elements $\langle m n\rangle$ expressible in the language defined as:

$$
\begin{array}{ll}
\langle m n\rangle & ::=\langle c m n\rangle \mid\langle u c m n\rangle \\
\langle c m n\rangle & ::=n \text { THEN }\langle u c m n\rangle \mid C \text { THEN }\langle u c m n\rangle \\
\langle u c m n\rangle & ::=O\left(n^{\prime}\right) \mid F\left(n^{\prime}\right)
\end{array}
$$

A use policy is just a set of meta-norms.

Definition 5. (Use Policy) A use policy $U P \subseteq M N$ is a set of meta-norms.

To exemplify, we formalise Peter's use policy $U P$ which specifies how Rachel may use his resource, where his resource is denoted with $p e, \mathrm{as}^{2}$ :

$$
\begin{aligned}
& U P=\left\{O _ { p e : r a } \left(s_{e n d} d_{a} a_{p e}\right.\right. \text { BEFORE leave_water_square) THEN } \\
& O\left(O_{\text {ra:pe }}\left(\text { pay }_{\text {ra }} \text { BEFORE tomorrow }\right)\right) \text {, } \\
& F\left(O_{p e: r a}\left(\text { send_data }_{p e} \text { BEFORE leave_water_square }{ }_{p e}\right)\right) \text {, } \\
& \text { battery_depleted }_{p e} \text { THEN F }\left(F _ { \text { pe:ra } } \left(\text { sensing_off }_{p e}\right.\right. \text { BEFORE } \\
& \text { gather_rain_data } \left.\left.\left.a_{p e}\right)\right)\right\}
\end{aligned}
$$

\subsection{Conflict}

Taking Rachel's request and Peter's use policy, we can intuitively see there are conflicts, which should be identified by the resource governance reasoner.

\footnotetext{
${ }^{2}$ More generalisable use policies are possible by extending the framework with variables. This would allow non-specific debtors and creditors to be specified, and the expression of meta-norms about norms where we are not concerned with exact terms, such as a specific deadline, e.g. 'it is forbidden to forbid me from turning my sensor off regardless of what the deadline is'.
} 
Example. (Part Three, Conflict) Rachel's request has been posed to Peter's agent in control of providing the resource. Rachel wants data sent before Peter's sensor leaves the water square area, however Peter has stated that he wants to be free to move. If Rachel's request is accepted then Peter will be prohibited from turning his sensor off, yet, he has stated that when his cellphone's battery is depleted he must be allowed to do so. Finally, once Peter has fulfilled an obligation to provide data, he demands to be paid before tomorrow, but Rachel's request does not include such an obligation.

The normative reasoner identifies sets of norms and meta-norms that hold simultaneously given some circumstances (a sequence of events), a request, and a use policy. Meanwhile the conflict-detector takes as input sets of simultaneously holding norms and meta-norms and identifies conflicts by comparing those sets.

The conflict detector identifies two types of conflict. The first type of conflict is where a meta-norm holds that obliges a norm to be detached, but that norm is not detached. Conversely, the second type of conflict is where a meta-norm holds that prohibits a norm from being detached, but that norm is detached.

We define conflict in terms of a set of norms and meta-norms that hold simultaneously. We assume the norms to be consistent and the meta-norms to be consistent, and focus on conflicts between norms and meta-norms.

Definition 6. (Conflict) Let $N^{\prime} \subset N$ be a set of unconditional norms and $M N^{\prime} \subset M N$ be a set of unconditional meta-norms, denoting all of the simultaneously detached norms and meta-norms for some circumstances. We say that there is a conflict between $N^{\prime}$ and $M N^{\prime}$ if either of the following holds:

$$
\left(n \in N^{\prime} \text { and } F(n) \in M N^{\prime}\right) \text { or }\left(n \notin N^{\prime} \text { and } O(n) \in M N^{\prime}\right)
$$

To exemplify, consider a sequence of events generated by the event sequence generator component, that can hypothetically happen after the request is accepted, where Peter enters the water square and then the battery on his phone is depleted. For this situation, assuming nothing else has happened, the set $D N$ is the set of all the norms from Rachel's request that are detached simultaneously with a set of all the meta-norms $D M N$ from Peter's use policy that are detached simultaneously:

$$
\begin{aligned}
& D N=\left\{F_{p e: r a}\left(\text { sensing_off }_{p e} \text { BEFORE gather_rain_data }{ }_{p e}\right)\right\} \\
& D M N=\left\{O \left(O_{\text {ra:pe }}\left(\text { pay }_{\text {ra }} \text { BEFORE tomorrow }\right),\right.\right. \\
& F\left(F_{p e: r a}\left(\text { sensing_off } f_{p e} \text { BEFORE gather_rain_data } a_{p e}\right)\right) \text {, } \\
& \left.F\left(O_{p e: r a}\left(\text { send_data }_{p e} \text { BEFORE leave_water_square }{ }_{p e}\right)\right)\right\}
\end{aligned}
$$

Both types of conflict are identified in this example when these sets of norms and meta-norms are compared. The first, is that a norm forbids turning the sensing off, yet a meta-norm is simultaneously detached that forbids such a prohibition. Similarly, a norm obliges Peter to send data before he leaves the water square, but such an obligation is prohibited by a detached meta-norm. Finally, a meta-norm obliges the obligation for payment to be provided, but such an obligation is not detached at the same time. 


\section{The Event Calculus Normative Model}

In this section we give the operational semantics for the detachment and termination of norms and meta-norms, and when they produce conflicts between a use policy and a request. We first re-introduce the Event Calculus (Section 5.1) which we subsequently use to define the operational semantics (Section 5.2).

\subsection{Event Calculus}

The Event Calculus is a logical-formalism specified by Kowalski and Sergot [11] for reasoning about events and their effects on which fluents hold and when. The Event Calculus provides an ontology of predicates (Table 1) for specifying in an Event Calculus theory the effects of events on initializing and terminating fluents, and what events happen at which time points (a narrative). The same ontology also provides predicates that specify, given the Event Calculus, an Event Calculus Theory and a narrative, which fluents hold at specific time intervals.

\begin{tabular}{ll}
\hline \multicolumn{1}{c}{ Predicate } & \multicolumn{1}{c}{ Meaning } \\
\hline broken_during $(P$, Start, End $)$ & $P$ is terminated between time points Start and End. \\
happens_at $(E, T)$ & The event $E$ happens at time $T$. \\
holds_at $(P, T)$ & The property $P$ holds at time $T$. \\
holds_for $(P, S t a r t, E n d)$ & The property $P$ holds from time points Start until End. \\
initially $(P)$ & The property $P$ holds at the first time point. \\
initiates_at $(E, P, T)$ & The event $E$ initiates the property $P$ at time $T$. \\
terminates_at $(E, P, T)$ & The event $E$ terminates the property $P$ at time $T$. \\
\hline
\end{tabular}

Table 1. The Event Calculus ontology of predicates

We choose the Event Calculus due to its modelling of inertial fluents and its efficient implementations [2,5]. Inertial fluents are required because we treat norms and meta-norms as fluents, and the informal notions of detached norms and meta-norms mean they continue to be detached until either their aim or deadline occurs. Efficiency is important, due to the time constraints that can be expected when accepting or rejecting a request.

Although many variations of the Event Calculus exist [14] we use the simple Event Calculus, where from here-on we usually omit the word simple. Whereas the full Event Calculus allows us to reason about events that terminate a fluent as implying the fluent held before the event occurred, we do not require this.

In the following, we give an axiomatisation of the Event Calculus adapted from [5] with the addition of a commonly used axiom for an initial state. Axioms are given as Prolog style horn-clauses with the head on the left and the tail on the right. Keeping with convention, symbols start with upper-case to denote variables and lower-case to denote constants. Since the Event Calculus explicitly deals with time, we assume an infinitely countable set of time instances $\mathbb{T}$ with 
typical element $t_{i}$ where $i \in \mathbb{N} \cup\{\infty\}$. The operators $<$ and $\leq$ are assumed to be specified for all members of the set $\mathbb{T}$, with the expected meaning. Finally, $\neg$, is interpreted as negation-as-failure, making the Event Calculus non-monotonic.

The first axiom, $e c 1$, states that any fluent stated to initially hold is initiated at the first time point.

$$
\text { initiates_at }(\operatorname{initially}(P), P, 0) \leftarrow \operatorname{initially}(P)
$$

The next two axioms specify the intervals fluents hold for. Axiom ec2 states that a fluent holds in an interval beginning immediately after the initiation event and ending at the termination event. Axiom ec 3 deals with the case where there is no terminating event for a fluent.

$$
\begin{aligned}
\text { holds_for }(P, \text { Start, End }) \leftarrow & \text { initiates_at }(\text { Ei, } P, \text { Start }) \wedge \\
& \text { terminates_at }(E t, P, \text { End }) \wedge \\
& \text { End }>\text { Start } \wedge \\
& \neg \text { broken_during }(P, \text { Start, End }) \\
\text { holds_for }\left(P, \text { Start }, t_{\infty}\right) \leftarrow & \text { initiates_at }(E i, P, \text { Start }) \wedge \\
& \neg \text { broken_during }\left(P, \text { Start }, t_{\infty}\right)
\end{aligned}
$$

Axiom ec4 states that a fluent is broken during an interval if a terminating event occurs during that interval. We specify the axiom such that it provides a 'weakinterpretation' of the initiates_at predicate [5], where the same initiation event occurring consecutively does not imply there was a terminating event in-between.

$$
\begin{gathered}
\operatorname{broken\_ during}(P, \text { Start, End }) \leftarrow \text { terminates_at }(E, P, T) \wedge \\
\text { Start }<T<E n d
\end{gathered}
$$

Finally, axiom ec5 provides a means to inspect the fluents that hold at specific time points as opposed to intervals.

$$
\text { holds_at }(P, T) \leftarrow \text { holds_for }(P, \text { Start, End }) \wedge \text { Start }<T \leq \text { End }
$$

Given the Event Calculus specification, the effects of events can be specified using the schemas ec6 for the initialisation of a fluent and $e c 7$, taken from [4].

$$
\begin{aligned}
& \text { initiates_at }(E, P, T) \leftarrow \text { happens_at }(E, T) \wedge \text { holds_at }\left(P_{1}, T\right) \\
& \wedge \ldots \wedge \text { holds_at }\left(P_{n}, T\right) \\
& \text { terminates_at }(E, P, T) \leftarrow \text { happens_at }(E, T) \wedge \text { holds_at }\left(P_{1}, T\right) \\
& \wedge \ldots \wedge \text { holds_at }\left(P_{n}, T\right)
\end{aligned}
$$

\subsection{Normative Evaluation and Conflict Checking}

Our normative evaluation and conflict checking semantics uses the Event Calculus for reasoning about which norms and meta-norms hold when, and when 
they conflict. The two resource governance reasoner components (see Figure 2), the Normative Evaluator, and the Conflict Checker, are defined as sets of Event Calculus rules.

In the following, we use the predicates $o / 4$ and $f / 4$ to respectively represent obligations and prohibitions, with the first two parameters respectively being the debtor and creditor, the third the aim, and the fourth the deadline event or $\perp$ to indicate no deadline. $o / 1$ and $f / 1$ are predicates representing meta-norms, where the parameter is a norm. We use the predicate ifthen $/ 2$ to represent conditional and unconditional norms and meta-norms, the first parameter is the condition or $T$ if it is unconditional, the second parameter is the norm or meta-norm.

As with work on social commitment modelling [4] we assume that two events cannot occur at the same time. We make an exception with respect to this for the event of a norm being detached, which can often occur at the same time as a non-detachment event and other norms being detached.

Normative Operational Semantics. The operational semantics of norms and metanorms correspond to the Normative Evaluator component (see Figure 2), specifying when a norm or meta-norm is and is not detached. The semantics are specified by defining axioms for the initiates_at/3 and terminates_at/3 Event Calculus predicates, which state an event (the first term) respectively detaches or terminates a norm or meta-norm (the second term) when the event happens (the last term). These axioms are defined with respect to the happens_at/2 predicate which describes when an event happens.

The first two axioms state that any unconditional norm holds initially.

$$
\text { initially }(o(D E, C R, A, D)) \leftarrow \text { ifthen }(\top, o(D E, C R, A, D))
$$

(Obl. Uncond. Norm Detachment)

$$
\operatorname{initially}(f(D E, C R, A, D)) \leftarrow \operatorname{ifthen}(\top, f(D E, C R, A, D))
$$

(Pro. Uncond. Detachment)

Obligatory meta-norms have different detachment semantics from norms. An unconditional obligatory meta-norm is detached initially only if it is not simultaneously satisfied with the detachment of a norm. We do not check if the norm it obliges is already detached, although this is certainly possible we take the meaning of an obligatory meta-norm to be that it obliges the detachment of a norm at the time it is itself detached. If the obligatory meta-norm is satisfied as soon as it is detached, then there is no conflict and so it will not be taken into account by the conflict checker.

$$
\text { initially }(o(\text { Norm })) \leftarrow \text { ifthen }(\top, o(\text { Norm })) \wedge \neg \text { initially }(\text { Norm })
$$

(Obl. MN Uncond. Detachment)

Unconditional prohibitory meta-norms, however, are detached regardless of whether the norm they prohibit is detached. Thus, their detachment follows the 
same form as norms, formulated in the next axiom.

$$
\text { initially }(f(\text { Norm })) \leftarrow \text { ifthen }(\top, f(\text { Norm })) \quad \text { (Pro. MN Uncond. Detachment) }
$$

The next two axioms, give the conditional detachment of norms, stating that when the condition of a conditional norm happens, the norm is detached.

$$
\begin{gathered}
\text { initiates_at }(C, o(D E, C R, A, D), T) \leftarrow \underset{i f t h e n}{ }(C, o(D E, C R, A, D)) \wedge \\
\text { happens_at }(C, T)
\end{gathered}
$$

(Obl. Cond. Detachment)

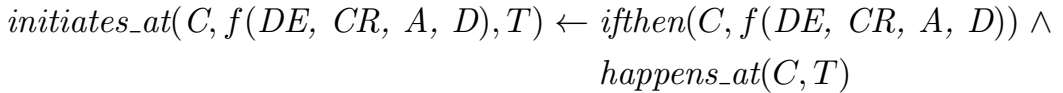

(Pro. Cond. Detachment)

The next axiom states that a conditional obligatory meta-norm is detached when its condition occurs, unless it is satisfied at the same time with the detachment of the norm it obliges (as with its unconditional variant). Again, if the obligatory meta-norm is detached and simultaneously satisfied, the conflict checker will not take it into account, because there is no conflict.

$$
\begin{aligned}
\text { initiates_at }(C 1, o(N o r m), T) \leftarrow & \operatorname{ifthen}(C 1, o(N o r m)) \wedge \text { happens_at }(C 1, T) \wedge \\
& \neg \text { initiates_at }(C 2, \text { Norm, T) }
\end{aligned}
$$

(Obl. MN Cond. Detachment)

Conditional prohibitory meta-norms, follow the same detachment semantics as conditional norms.

$$
\text { initiates_at }(C, f(N o r m), T) \leftarrow \text { ifthen }(C, f(N o r m)) \wedge \text { happens_at }(C, T)
$$

(Pro. MN Cond. Detachment)

We treat the detachment of a norm as an event, the event of the norm being imposed on an agent. This is required for the detachment of meta-norms that are conditional on the event of a norm being detached and the satisfaction of obligatory meta-norms which is the event of a norm being detached.

$$
\begin{gathered}
\text { happens_at }(o(D E, C R, A, D), T) \leftarrow \underset{i f t h e n}{ }(C, o(D E, C R, A, D)) \wedge \\
\text { happens_at }(C, T)
\end{gathered}
$$

(Obl. Detachment Event)

$$
\begin{aligned}
& \text { happens_at }(f(D E, C R, A, D), T) \leftarrow \text { ifthen }(C, f(D E, C R, A, D)) \wedge \\
& \text { happens_at }(C, T)
\end{aligned}
$$

(Pro. Detachment Event)

We now turn our attention to the termination of detached norms. A detached obligation is terminated if its aim is achieved, whilst a detached prohibition is terminated if its deadline occurs. Thus, although we do not explicitly model 
violations, under these semantics a norm persists after it is violated until it is fulfilled. Alternative semantics can be accommodated for in the future.

$$
\text { terminates_at }(A, o(D E, C R, A, D), T) \leftarrow \text { happens_at }(A, T)
$$

(Obl. Aim Termination)

$$
\text { terminates_at }(D, f(D E, C R, A, D), T) \leftarrow \text { happens_at }(D, T)
$$

(Pro. Deadl. Termination)

As with obligatory norms, obligatory meta-norms are terminated when their aim (the detachment of a norm) occurs:

$$
\text { terminates_at }(\text { Norm, o(Norm }), T) \leftarrow \text { happens_at }(\text { Norm, T) }
$$

(Obl. MN Aim Termination) Prohibitory meta-norms, like their norm counterparts, are not terminated when their aim occurs (a norm they prohibit is detached). Thus, due to not having a deadline, they are not terminated at all.

Conflict Detection Semantics. The conflict detection semantics correspond to the Conflict Checker component (see Figure 2). The semantics are given as axioms for the predicate conflict/3, which states a meta-norm is causing conflict (the first term), from when (the second term) and until when (the last term). As conceptually defined in Definition 6 Conflict is determined based on which norms and meta-norms hold for the same period of time, this is given by the results of the Normative Operational Semantics.

The first type of conflict occurs when a meta-norm obliges the detachment of a norm and that norm is not detached. If this is the case, then the obligatory meta-norm will hold for some time until it is satisfied. We do not need to check whether an obligatory meta-norm holds and the norm it obliges does not, the obligatory meta-norm holding already implies this.

$$
\text { conflict }(o(\text { Norm }), \text { Start }, \text { End }) \leftarrow \text { holds_for }(o(\text { Norm }), \text { Start, End })
$$

(Obl. MN Conflict)

The final axiom states that given two overlapping intervals where a norm holds and a prohibitory meta-norm holds, there is a conflict if the norm is prohibited by the meta-norm. We assume the predicates minimum $\left(T, T^{\prime}, M i n\right)$ and $\operatorname{maximum}\left(T, T^{\prime}, \operatorname{Max}\right)$ are defined with the meaning that for two time points $T, T^{\prime} \in \mathbb{T}$, the predicate minimum/3 holds if Min is the minimum of the two time points, and the predicate maximum $/ 3$ holds if Max is the maximum of the two time points. This allows us to define what an overlapping period is.

$$
\begin{aligned}
\text { conflict }(f(\text { Norm }), \text { Start }, \text { End }) \leftarrow & \text { holds_for }(f(\text { Norm }), \text { MNStart, MNEnd }) \wedge \\
& \text { holds_for }(\text { Norm, NStart, NEnd }) \wedge \\
& \text { maximum }(\text { NStart, MNStart, Start }) \wedge \\
& \text { minimum }(\text { NEnd, MNEnd, End }) \wedge \text { Start }<\text { End }
\end{aligned}
$$

(Pro. MN Conflict) 


\section{An illustration}

We can now show how our formalism works for conflict detection with respect to the running example of Rachel's request and Peter's use policy. First, we assume the following event sequence produced by a sequence generator component. Naturally, not all events cause conflicts between the use policy and request, and different sequences can produce different results:

$$
\begin{aligned}
& E 1=h a p p e n s \_a t(\text { enter_water_square } \\
& \text { pe } \\
& E 3=\text { happens_at }\left(\text { gather_rain_data } a_{p e}, 3\right), E 4=\text { happens_at }\left(\text { battery_depleted }_{p e}, 2\right),
\end{aligned}
$$

From these events, we can infer the following norm detachment events:

$$
\begin{aligned}
& E 5=\text { happens_at }\left(O_{p e: r a}\left(\text { gather_rain_data } a_{p e} \text { BEFORE send_data } a_{p e}\right), 1\right), \\
& E 6=\text { happens_at }\left(O_{p e: r a}\left(\text { send_data }_{p e} \text { BEFORE leave_water_squar } e_{p e}\right), 1\right), \\
& E 7=\text { happens_at }\left(F_{r a: p e}\left(\text { sensing_off } f_{p e} \text { BEFORE gather_rain_data } a_{p e}\right), 1\right)
\end{aligned}
$$

For these events, Figure 3 depicts which fluents hold and when, and the conflicts.

$$
\begin{aligned}
& O_{p e: r a}\left(\text { gather_rain_data }{ }_{p e} \text { BEFORE send_data }{ }_{p e}\right) \\
& O_{p e: r a}\left(\text { send_data }{ }_{p e} \text { BEFORE leave_water_square }{ }_{p e}\right) \\
& F_{p e: r a}\left(\text { sensing_off pe BEFORE gather_rain_data } a_{p e}\right) \\
& \left.F\left(\underset{O_{p e: r a}\left(\text { send_data }_{p e}\right. \text { BEFORE leave_water_square }}{\stackrel{\longrightarrow}{\longrightarrow}}\right)\right) \\
& \text { E60 } \left.\left.\underset{O\left(O_{\text {ra:pe }}(\text { pay }\right.}{ } \text { Ba BEORE tomorrow }\right)\right)
\end{aligned}
$$

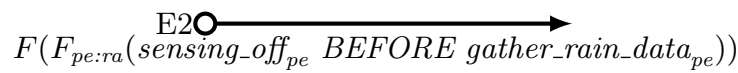

$$
\begin{aligned}
& \text { conflict }\left(O\left(O_{\text {ra:pe }}(\text { pay } \text { ra } \text { BEFORE tomorrow })\right)\right) \\
& \operatorname{conflict}\left(F\left(F_{p e: r a}(\overbrace{\text { sensing_off }} \text { BEFORE gather_rain_data }{ }_{p e})\right)\right) \\
& \left.\operatorname{conflict}\left(F\left(O_{\text {pe:ra }} \underset{\left(\text { send_data }_{p e}\right. \text { BEFORE leave_water_square }}{p e}\right)\right)\right)
\end{aligned}
$$

Fig. 3. An example with several conflicts. — indicates the interval a fluent holds for, $\mathbf{O}-\mathbf{O}$ is a terminated interval, whilst $\mathbf{O} \rightarrow$ is an interval that continues forever.

As we intuitively expect, there is a conflict because Peter is obliged to send data, but Rachel is not obliged to pay him. Another conflict occurs because 
Peter is forbidden from turning his device off, but because his phone's battery has become low he wants to maintain this right. Finally Peter is obliged to send data before leaving the water square, but such an obligation is forbidden.

\section{Conclusions}

In this paper our main contribution was a novel temporal event-based reasoner for determining if there are conflicts between a request to use a resource and use policy governing a resource's usage. This allows owners of resources to maintain autonomy over how, when and to whom their resources are used. Taking the notion of a request for the use of a resource as a set of norms, we gave a representation of a use policy, specifying what norms a request should and should not impose on a resource under some circumstances, as a set of meta-norms. Our reasoner detects whether the requirements of a use policy are met by a request or if the request has conflicts. Our proposal is particularly robust because the operational semantics ensures a conflict is detected between a norm and a meta-norm only based on whether they can be detached simultaneously.

There are many interesting avenues for future work, we go over some of the most immediate extensions here. By extending the representation of meta-norms to include an 'Or Else' option, such as 'you should not obligate me to do X, but if you do then you should not forbid me to do Y' we will be able to investigate computing a partial ordering of ideal and sub-ideal requests. This can facilitate better decision making in a resource governing agent, such as which requests are the best to accept when there are many offered, or support negotiation with a qualitative model of preferences.

Finally, in our proposal, we limit conditions, aims and deadlines to propositions. This makes sense since it allows us to express concrete norms in a request. For future work we can extend the representation languages to make use of variables in first-order logic. This will allow a user to express meta-norms such as 'you are prohibited from obligating me to do anything' and even meta-norms with constraints such as 'you are forbidden from obligating me to pay you anything over $€ 10$ '.

\section{Acknowledgements}

This work is supported by the SHINE project of TU Delft ${ }^{3}$, funded by the Delft Institute for Research on ICT (DIRECT).

\section{References}

1. G. Andrighetto, G. Governatori, P. Noriega, and L. van der Torre. Normative Multi-Agent Systems. Dagstuhl Follow-Ups, 4, 2013.

\footnotetext{
${ }^{3}$ http://direct.tudelft.nl/shine-117.html
} 
2. A. Artikis, M. Sergot, and G. Paliouras. Run-time composite event recognition. In Proceedings of the 6th ACM International Conference on Distributed Event-Based Systems, pages 69-80, New York, New York, USA, 2012. ACM Press.

3. G. Boella and L. van der Torre. Permissions and obligations in hierarchical normative systems. In Proceedings of the 9th International Conference on Artificial Intelligence and Law, pages 109-118, 2003.

4. F. Chesani, P. Mello, M. Montali, and P. Torroni. Representing and monitoring social commitments using the event calculus. Autonomous Agents and Multi-Agent Systems, 27(1):85-130, June 2012.

5. L. Chittaro and A. Montanari. Efficient temporal reasoning in the cached event calculus. Computational Intelligence, 12(3), 1996.

6. V. Dignum. A Model for Organizational Interaction: Based on Agents, Founded in Logic. PhD thesis, University of Utrecht, Utrecht, The Netherlands, 2003.

7. D. Gabbay, J. Horty, X. Parent, R. van der Meyden, and L. van der Torre, editors. Handbook of Deontic Logic and Normative Systems vol. 1. 2013.

8. R. Ganti, F. Ye, and H. Lei. Mobile crowdsensing: current state and future challenges. IEEE Communications Magazine, 49(11):32-39, Nov. 2011.

9. G. Governatori. Representing business contracts in RuleML. International Journal of Cooperative Information Systems, 14(2-3):181-216, 2005.

10. J. Hübner, J. S. Sichman, and O. Boissier. A model for the structural, functional, and deontic specification of organizations in multiagent systems. LNCS, 2507:118$128,2002$.

11. R. Kowalski and M. Sergot. A logic-based calculus of events. New Generation Computing, 4(1):67-95, 1986.

12. F. L. y. López, M. Luck, and M. D'Inverno. A normative framework for agent-based systems. Computational and Mathematical Organization Theory, 12(2-3):227-250, 2006.

13. X. Parent and L. van der Torre. Input/output Logic. In D. Gabbay, J. Horty, X. Parent, R. van der Meyden, and L. van der Torre, editors, Handbook of Deontic Logic and Normative Systems Vol. 1, pages 499 - 544. 2013.

14. M. Shanahan. The event calculus explained. Artificial Intelligence Today, pages 409-430, 1999.

15. M. B. van Riemsdijk, L. A. Dennis, M. Fisher, and K. V. Hindriks. Agent Reasoning for Norm Compliance A Semantic Approach. In Proceedings of the 12th International Conference on Autonomous Agents and Multiagent Systems (AAMAS 2013), pages 499-506, Saint Paul, Minnesota, USA, 2013.

16. H. Wansing. Nested deontic modalities: Another view of parking on highways. Erkenntnis, 49(2):185-199, 1998.

17. P. Yolum and M. Singh. Reasoning about commitments in the event calculus: An approach for specifying and executing protocols. Annals of Mathematics and Artificial Intelligence, 42(1-3):227-253, 2004.

18. P. Yolum and M. P. Singh. Flexible Protocol Specification and Execution : Applying Event Calculus Planning using Commitments. In The First International Joint Conference on Autonomous Agents and Multiagent Systems: Part 2, pages 527-534, 2002. 